\title{
CORRELAÇÃO ENTRE OS DIAGNÓSTICOS CITOPATOLÓGICOS E HISTOPATOLÓGICOS DAS LESÕES DA MUCOSA ORAL APÓS A PUNÇÃO ASPIRATIVA POR AGULHA FINA
}

\author{
CORRELATIONSHIP OF CITOPATHOLOGY AND HISTOPATHOLOGY ON ORAL \\ MUCOSA LESIONS AFTER FINE NEEDLE ASPIRATION
}

\author{
Tatiana Ferreira Robaina1; Antonio Carlos de Azeredo Coutinho² ; Carla Peixoto Valladares; \\ Eliane Pedra Dias"; Simone de Queiroz Chaves Lourenço ${ }^{5}$
}

\begin{abstract}
RESUMO: Objetivo. Fazer um estudo retrospectivo, correlacionando os diagnósticos citopatológicos obtidos por punção aspirativa por agulha fina (PAAF) com os diagnósticos histopatológicos de lesões da região bucomaxilofacial, de pacientes que foram atendidos no Serviço de Cirurgia e Traumatologia Bucomaxilofacial do Hospital Universitário Antonio Pedro/UFF, entre maio de 1999 e maio de 2004. Método. Foram analisados os laudos cito e histopatológicos emitidos pelo Serviço de Anatomia Patológica (SAP-HUAP), de quarenta e cinco pacientes submetidos à a punção aspirativa por agulha fina (PAAF) por apresentar lesão na região bucomaxilofacial. Resultados. Foi obtida uma precisão diagnóstica da citopatologia a partir da PAAF de 77,8\% (35 casos), e especificidade e sensibilidade da técnica de $100 \%$, não havendo nenhum resultado falso-positivo e falso-negativo. Conclusão. A citopatologia realizada a partir da PAAF é um método diagnóstico inicial eficiente, de baixo custo, praticamente indolor e rápido de ser realizado, que contribui para a identificação da natureza da lesão proporcionando um melhor planejamento terapêutico (Rev. Col. Bras. Cir. 2007; 34(5): 285-289).
\end{abstract}

Descritores: Biópsia por agulha; Boca/ lesões; Mucosa bucal; Doenças da boca/ diagnóstico.

\section{INTRODUÇÃO}

A tumefação de estruturas intra e extra-orais na região da cabeça, bem como nas regiões cervicais, é quei$x a$ freqüente de pacientes que procuram os serviços de cirurgia bucomaxilofacial e cirurgia de cabeça e pescoço. Em função da variada natureza dos tecidos desta região, muitas vezes é difícil estabelecer um diagnóstico preciso e definir uma conduta adequada de procedimentos, havendo assim a necessidade de ser realizada uma biópsia da lesão para que, com o resultado histopatológico, seja iniciado o tratamento.

A citopatologia feita a partir da aspiração por punção com agulha fina (PAAF) destas lesões tem se mostrado um método auxiliar importante, fornecendo, na maioria das vezes, um diagnóstico rápido e preciso ${ }^{1}, 2$. Ela não substitui a histopatologia para o diagnóstico definitivo, mas pode orientar o profissional para um melhor direcionamento da conduta terapêutica, diferenciando a natureza da lesão em inflamatória e neoplásica, benigna ou maligna ${ }^{3},{ }^{4,5,6}$. A utilização desta técnica auxiliar de diagnóstico justifica-se uma vez que um bom prognóstico está diretamente relacionado ao diagnóstico precoce e a uma conduta terapêutica adequada.

Desde os anos 70, a PAAF ganhou adeptos ${ }^{7}$, embora muitas opiniões contrárias existam desde seu surgimento, e a técnica ainda hoje seja motivo de discussão. Em sua maioria, os trabalhos afirmam que é uma técnica bastante eficaz e precisa no diagnóstico de vários tumores, principalmente os de glândulas salivares, onde seu emprego obtém bons resultados. A maioria dos trabalhos em glândulas salivares obteve uma precisão diagnóstica que variou de $83 \%$ a $100 \% \%^{2-6}$.É uma técnica rápida, praticamente indolor, de custo baixo e de fácil execução, sem relatos de complicações após sua aplicação, sendo bem aceita pelos pacientes, inclusive pelos pediátricos ${ }^{1,7}$. Como acontece no emprego de qualquer outra técnica, há limitações e contra-indicações, bem como a possibilidade de resultados falso-positivos e falso-negativos ${ }^{6,8}$. Este é um fato importante, visto que pode levar o profissional a planejar um tratamento inadequado, particularmente problemático quando se suspeita de lesões malignas. Há uma variação de resultados falso-positivos de $0 \%$ a $14 \%$ e falso-negativos de $0 \%$ a $16 \%^{2,9,10}$. Apesar de proporcionar alta

1. Cirurgiã-Dentista; Especialista em Metodologia do Ensino Superior; Mestre em Patologia, UFF.

2. Cirurgião-Dentista; Professor Assistente do Departamento de Cirurgia Bucomaxilofacial da Universidade Federal Fluminense - UFF, Niterói, RJ.; Mestre em Patologia, UFF, Niterói - RJ..

3. Cirurgiã-Dentista; Especialista em Estomatologia, UFRJ; Mestre em Patologia, UFF, Niterói - RJ.

4. Médica; Doutora em Anatomia Patológica, UFF, Niterói - RJ; Professora Adjunta da Universidade Federal Fluminense, UFF, Niterói - RJ; Coordenadora do Programa de Pós-Graduação em Patologia, UFF, Niterói, RJ.

5. Cirurgiã-Dentista; Doutora em Patologia Bucodental; Professora Adjunta do Departamento de Patologia da Universidade Federal Fluminense, UFF, Niterói, RJ

Recebido em 28/01/2007

Aceito para publicação em 30/03/2007

Conflito de interesses:nenhum

Fonte de financiamento:nenhuma

Trabalho realizado no Serviço de Cirurgia e Traumatologia Bucomaxilofacial do Hospital Universitário Antonio Pedro/UFF. 
confiabilidade, justamente por este fato a utilização da PAAF não descarta a indicação de biópsia, principalmente em casos de suspeita de linfomas ou de resultados inespecíficos ${ }^{11},{ }^{12}$. Apesar disto, a maioria dos estudos concorda que a citopatologia por PAAF é um recurso importante para o planejamento do tratamento, uma vez que não se pode baseá-lo somente no diagnóstico clínico ${ }^{1,7}$. Muitos autores relatam que a PAAF tem grande valor no estudo e no tratamento de lesões da cabeça e pescoço, principalmente para distinguir uma doença neoplásica de uma inflamatória ${ }^{2,13}$.

Uma possível solução para evitar uma PAAF malsucedida é a utilização de recursos como a radiografia e a ultra-sonografia para guiar este procedimento, diminuindo assim a possibilidade de erro durante a sua execução ${ }^{14,15}$. O treinamento do profissional que a realiza e o cuidadoso processamento das lâminas também são essenciais para o sucesso da técnica ${ }^{16}$.

O estudo comparativo entre os resultados obtidos na citopatologia por PAAF e os resultados dos laudos obtidos pela histopatologia é um importante meio de avaliação da precisão diagnóstica deste método. Este trabalho foi realizado na tentativa de contribuir com mais informações sobre a validade desta técnica, constituindo-se de um estudo retrospectivo, com a finalidade de correlacionar os diagnósticos citopatológicos obtidos por PAAF com os diagnósticos histopatológicos de lesões da região bucomaxilofacial, e analisar a sensibilidade e especificidade da PAAF comparando os diagnósticos citopatológicos e histopatológicos.

\section{MÉTODO}

Foi realizado um estudo retrospectivo, onde a amostra foi estabelecida a partir da seleção de pacientes atendidos no Ambulatório de Cirurgia e Traumatologia Bucomaxilofacial, com laudos citopatológicos e histopatológicos emitidos pelo Serviço de Anatomia Patológica do Hospital Universitário Antônio Pedro (HUAP/UFF), entre maio de 1999 e maio de 2004. Todos os casos selecionados referiam-se a pacientes que apresentaram lesões na região bucomaxilofacial com laudos diagnósticos citopatológicos e histopatológicos obtidos das mesmas lesões e já arquivados.

Foram descartados os casos que tinham mais de uma punção aspirativa da mesma lesão, bem como aqueles com informações incompletas, perfazendo um total de 54 casos com todas as informações necesssárias. Nesta seleção não houve preocupação quanto a idade, o gênero e a etnia dos pacientes. Todos os prontuários que se encontravam completos, ou seja, com o laudo cito e histopatológico e respectivas lâminas, foram comparados. As lâminas correspondentes aos prontuários foram revistas por dois anatomo-patologistas a fim de obter a confirmação do diagnóstico e assim realizar a análise da precisão da citopatologia realizada por PAAF das lesões. Todas as lâminas de citopatologia estavam coradas pelo método de Papanicolaou e as de histopatologia pelo método de Hematoxilina e Eosina (HE).

A sensibilidade foi interpretada em relação a quantidade de falsos-positivos, ou seja a a medida da capacidade do método de predizer a doença para aqueles casos que a apre- sentam ${ }^{17}$. A especificidade como a quantidade da falsos-negativos, ou seja, a capacidade do método de predizer a ausência de doença. A acurácia do método diz respeito aos resultados verdadeiros, ou seja, a proporção de predições corretas.

\section{RESULTADOS}

Do total de 54 prontuários selecionados por estarem com documentação completa, nove $(16,7 \%)$ deles tinham material proveniente da PAAF insuficiente para análise citopatológica. Sendo assim, o diagnóstico comparativo só pôde ser estabelecido em 45 casos $(83,3 \%)$.

Dentre os 45 prontuários com material suficiente para análise citopatológica, identificou-se 35 (77,8\%) com coincidência entre os diagnósticos citopatológico e histopatológico; em cinco $(11,1 \%)$ não houve correspondência entre os diagnósticos e em cinco $(11,1 \%)$ casos, o diagnóstico não foi correspondente, mas foi excludente de malignidade, identificando corretamente a natureza da lesão.

Obtivemos correspondência diagnóstica precisa histo e citopatológica em 35 prontuários. Isso equivale a uma precisão diagnóstica da PAAF de 77,8\%. Dos 35 com absoluta correspondência diagnóstica, foram identificados três $(8,5 \%)$ como lesões malignas - todos eram carcinoma de células escamosas. A técnica não forneceu diagnóstico preciso em 10 pacientes, porém vale na exclusão de malignidade em todos os casos e também no diagnóstico de lesões benignas, o que equivale a uma sensibilidade e uma especificidade de $100 \%$. Não houve nenhum resultado falso-negativo para malignidade e nenhum falso-positivo. Diante disso, a acurácia do método foi de $100 \%$.

As lesões benignas, que corresponderam ao total de $32(91,4 \%)$ casos entre os 35 casos que tiveram correspondência diagnóstica, se dividiram nas seguintes lesões: oito (25\%) adenomas pleomórficos (Figuras 1, 2, 3, 4), seis $(18,7 \%)$ casos de sialodenite crônica, cinco $(15,6 \%)$ cistos radiculares, quatro $(12,5 \%)$ cistos ósseos traumáticos, dois $(6,2 \%)$ fenômenos de extravasamento de muco, dois $(6,2 \%)$ cistos ósseos aneurismáticos, um $(3,1 \%)$ linfonodo reacional, um $(3,1 \%)$ granuloma de células gigantes, um $(3,1 \%)$ granuloma periapical, um $(3,1 \%)$ osteomielite, um $(3,1 \%)$ Tumor de Warthin.

\section{DISCUSSÃO}

Entre os 54 casos selecionados como compor a amostra, $45(83,3 \%)$ puderam ser analisados, pois possuíam material suficiente nas lâminas, proporcionando boas condições diagnósticas. No entanto, consideramos que esta é uma porcentagem que pode ser melhorada se o profissional que realiza a PAAF e o técnico que processa as lâminas forem bem treinados ${ }^{5,13,16}$, este número pode ser aumentado significativamente, uma vez que na literatura, os mesmos autores citam até $85 \%$ de aproveitamento das lâminas processadas. Também o profissional que faz a interpretação do exame citopatológico por PAAF deve ter experiência e ser criterioso. Em todos estes estudos houve uma porcentagem de lâminas que não puderam ser lidas. É importante que seja esclarecido o que impossibilitou esta leitura a fim de que outros estudos 


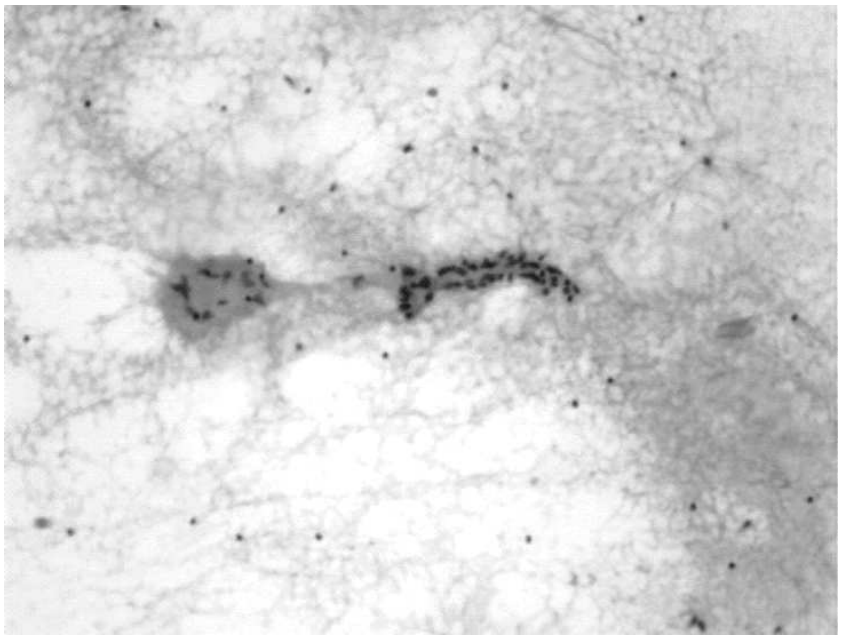

Figura 1 - Citopatologia: células semelhantes a células ductais, sugerindo diagnóstico de adenoma pleomórfico (Papanicolaou, 10X).

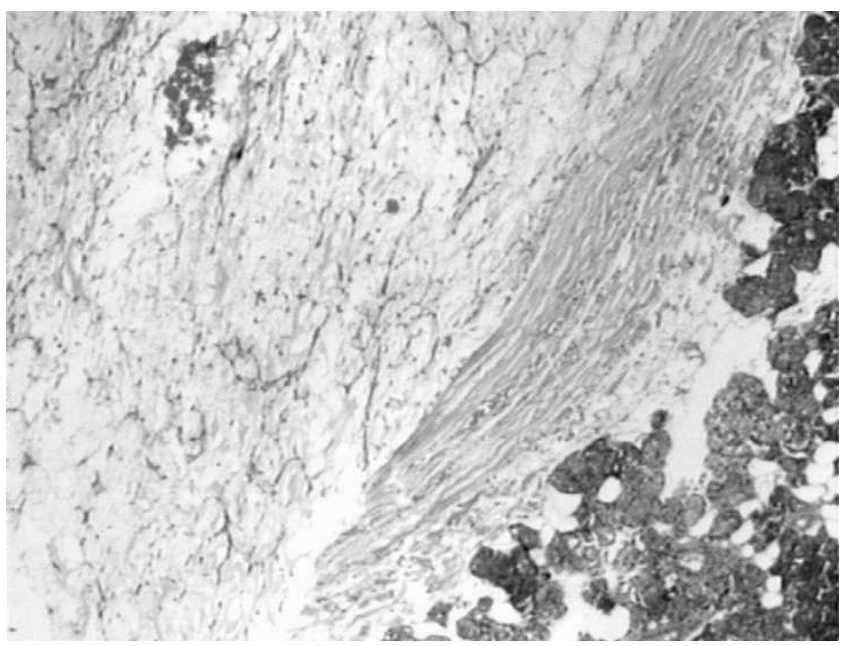

Figura 3 - Histopatologia: confirmação do diagnóstico de Adenoma Pleomórfico (HE, 10X).

tenham parâmetros para comparar seus resultados, podendo, assim, analisar suas falhas ${ }^{18}$.

APAAF é uma técnica simples, precisa, de baixo custo, praticamente indolor, que pode ser indicada a qualquer tipo de lesão aparente em qualquer paciente ${ }^{19}$. Como visto no nosso estudo, a PAAF pode ser aplicada a diferentes lesões e em diferentes tecidos com razoável sucesso e pode também ser utilizada como recurso diagnóstico auxiliar inclusive em lesões que acometem crianças ${ }^{9}$.

A citopatologia a partir da PAAF é pouco invasiva e consiste num método importante para um primeiro diagnóstico. De acordo com nosso estudo, tem boa precisão diagnóstica $(64,8 \%)$, tendo um valor sem igual para uma melhor condução terapêutica, estando em concordância com diversos trabalhos publicados. 2,3,10,20, 21

Em relação aos tumores de glândulas salivares tivemos cinco adenomas pleomórficos, que tiveram total correlação histocitopatológica. De acordo com este resultado, a PAAF torna-se de imprescíndivel realização quando há suspeita desta lesão. Considerando ainda tratar-se de uma lesão altamente recidivante e agressiva, é absolutamente necessá-

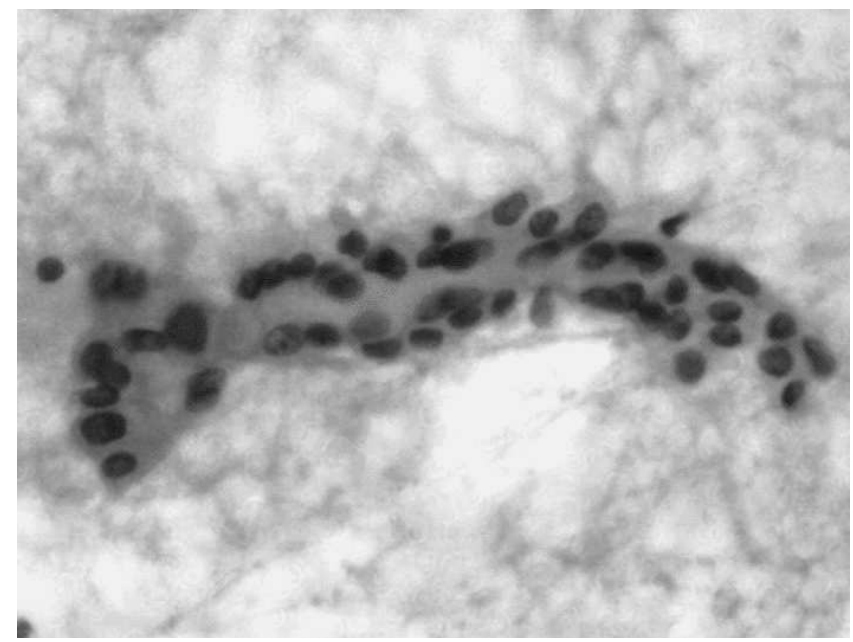

Figura 2 - Citopatologia: células semelhantes a células ductais, sugerindo diagnóstico de adenoma pleomórfico (Papanicolaou, 40X).

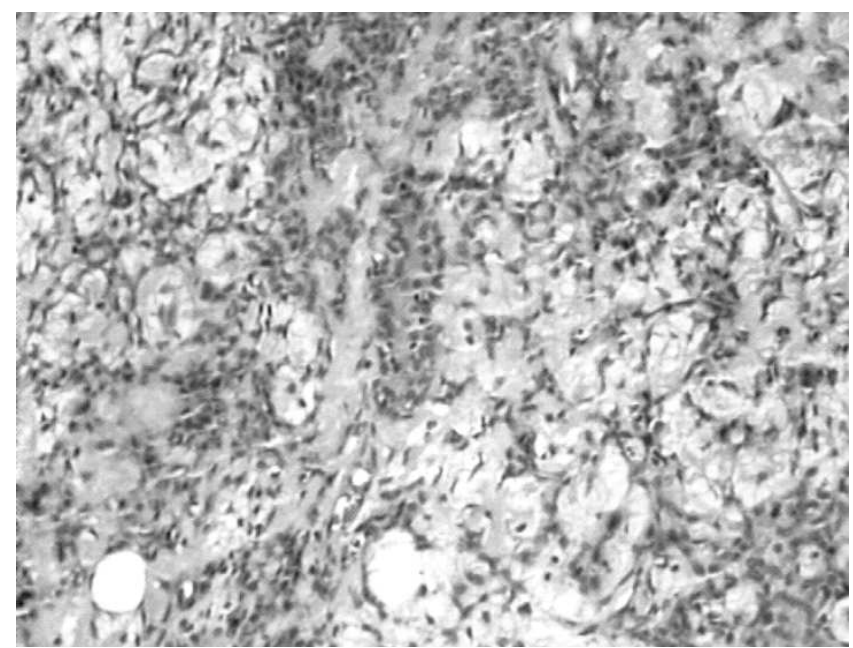

Figura 4 - Histopatologia: confirmação do diagnóstico de Adenoma Pleomórfico (HE, 40X).

rio um diagnóstico preciso e precoce a fim de dar ao paciente um tratamento adequado $3,5,22$.

Dentre os nove $(16,6 \%)$ casos que tiveram material insuficiente para a análise citopatológica, sete $(77,7 \%)$ eram de glândulas salivares e duas $(33,3 \%)$ de lesões intra-ósseas. Este fato deve ser analisado cautelosamente, pois pode significar imperícia do profissional que fez a PAAF, uma vez que lesões glandulares costumam ter maior abundâcia de material a ser extraído.

Consideramos que a PAAF tem grande valor para a diferenciação de lesões malignas e benignas, sendo essencial para uma terapia mais conservadora. Além disto, é muito indicada quando as lesões se encontram próximas a acidentes anatômicos nobres, evitando danos desnecessários a estas estruturas.

Neste estudo não foi encontrado nenhum resultado falso-negativo e nenhum falso-positivo. Muitos autores obtiveram em seus trabalhos alguns destes resultados, no entanto, isto não aconteceu em nosso estudo, provavelmente por termos trabalhado com uma amostra menor que a de todos estes autores ${ }^{3,5,10,16}$. Obtivemos uma sensibilidade de $100 \%$, 
pois todos os tumores malignos foram diagnosticados corretamente como malignos pela citopatologia, coincidindo com a literatura ${ }^{7}$. Em relação às lesões de origem inflamatória, todas foram corretamente diagnosticadas pela citopatologia como não-malignas, e foi indicada sua possível origem. Obtivemos, então, uma especificidade de $100 \%$.

Todas as lesões malignas encontradas no nosso tabalho foram diagnosticadas como carcinoma de células escamosas e tiveram total correspondência diagnóstica citohistopatologicamente. Embora fosse um amostra pequena (três casos), ressaltamos que a citopatologia a partir da PAAF é um método que pode ser realizado quando desconfiamos deste tipo de lesão, pois é rápido e de ótima precisão diagnóstica, colaborando para o planejamento da conduta terapêutica deste paciente. Bons resultados foram obtidos com esta manobra em lesões malignas ${ }^{10,23}$. No entanto, esta técnica é uma opção e não dispensa outras técnicas como um raspado ou a biópsia sacabocado.

A citopatologia feita a partir da PAAF é um método diagnóstico auxiliar rápido, que quando inconclusivo pode ser repetido sem danos para o paciente ou complicações pós-operatórias, e não impede que se parta imediatamente para a biópsia tradicional. Quando nos deparamos com amostras insatisfatórias devemos procurar saber se houve algum erro e tentar corrigi-lo para melhorar a precisão deste método.

De acordo com os resultados obtidos no nosso estudo, recomendamos que a citopatologia a partir da PAAF deva ser indicada para o diagnóstico inicial de lesões da região bucomaxilofacial, pois há grande precisão diagnóstica, facilitando e orientando o planejamento terapêutico do profissional.

De acordo com os resultados obtidos em nosso estudo, concluímos que a citopatologia realizada a partir da punção aspirativa por agulha fina das lesões da região bucomaxilofacial é um método eficaz, de baixo custo e relativamente indolor quando comparada com a biópsia convencional, embora não a substitua. Ainda assim, por apresentar um grande índice de precisão diagnóstica, é um exame válido como direcionador do diagnóstico inicial.

\begin{abstract}
Background. To establish a prospective study to verify the correlation between the cytopathologic diagnosis obtained through FNA and the hystopathologic diagnosis of the lesions from the maxillofacial region of patients at the Buco-Maxilofacial Surgery and Traumatology Service. Method. Analysis of the results from cytologic and histopathological diagnosis issued by the Pathologic Anatomy Service of the Antônio Pedro University Hospital (UFF, Niterói-RJ, Brazil). Forty-five patient who underwent FNA followed by incisional or excisional biopsy, were selected. Cases in which information was incomplete were excluded. Results. The total number of cases selected was 45. The diagnostic accuracy obtained was of $77.8 \%$ (35) in FNA cytopathology, with a $100 \%$ in specificity and sensibility. Conclusion. The cytopathology performed through FNA is an efficient initial diagnostic method, with low cost, painless, and quickly accomplished, which helps the identification of the lesion's nature and allows the professional a more accurate evaluation for the surgical or therapeutic approach.
\end{abstract}

Key words: Biopsy, needle; Mouth/lesions; Mouth mucosa; Mouth diseases/ diagnosis.

\section{REFERÊNCIAS}

1. Layfield LJ, Tan P, Glasgow BJ. Fine-needle aspiration of salivary gland lesions. Comparison with frozen sections and histologic findings. Arch Pathol Lab Med. 1987;111(4):346-53.

2. Gonçalves SLM. Estudo comparativo entre a citopatologia obtida pela punção aspirativa por agulha fina e a histopatologia em tumores da região submandibular [dissertação]. Rio de Janeiro (RJ): Universidade Federal do Rio de Janeiro; 1998.

3. Zbaren P, Schar C, Hotz M, Loosli H. Value of fine-needle aspiration cytology of parotid gland masses. Laryngoscope. 2001;111(11 Pt 1):1989-92.

4. Amedee RG, Dhurandhar NR. Fine-needle aspiration biopsy. Laryngoscope. 2001;111(9):1551-7.

5. Cohen EG, Patel SG, Lin O, Boyle JO, Kraus DH, Singh B, Wong RJ, Shah JP, Shaha AR. Fine-needle aspiration biopsy of salivary gland lesions in a selected patient population. Arch Otolaryngol Head Neck Surg. 2004;130(6):773-8.

6. Das DK, Petkar MA, Al-Mane NM, Sheikh ZA, Mallik MK, Anim JT. Role of fine needle aspiration cytology in the diagnosis of swellings in the salivary gland regions: a study of 712 cases. Med Prin Pract. 2004;13(2):95-106.
7. Feldman PS, Kaplan MJ, Johns ME, Cantrell RW. Fine-needle aspiration in squamous cell carcinoma of the head and neck. Arch Otolaryngol Head Neck Surg. 1983;109(11):234-7.

8. Livingstone C. Fine needle aspiration cytology. $2^{\text {nd }}$ ed. New York: Editorial Resources Unit; 1992.

9. Liu ES, Bernstein JM, Sculerati N, Wu HC. Fine needle aspiration biopsy of pediatric head and neck masses. Int J Pediatr OtoI. 2001;60(2):135-40.

10. Draper MR, Pfleiderer AG, Smith W. Assessment of a cytology grading system for head and neck masses. Clin Otolaryngol Allied Sci. 2003;28(1):34-8.

11. Ramzy I, Rone R, Schultenover, SJ, Buhaug J. Lymph node aspiration biopsy. Diagnostic reliability and limitations: an analysis of 350 cases. Diagn Cytopathol. 1985;1(1):39-45.

12. Layfield LJ, Reichman A. Fine needle aspiration cytology: utilization in pediatric pathology. Dis Markers. 1990;8:(6):301-15.

13. El-Hag IA, Chiedozi LC, Al Reyees FA, Kollur SM. Fine needle aspiration cytology of head and neck masses. Seven years' experience in a secondary care hospital. Acta Cytol. 2003;47(3):387-92.

14. Yoon C, Lufkin R. Guided aspiration cytology. Clin MRI. 1991;1:113. 
15. Dejmek A, Lindholm K. Fine needle aspiration biopsy of cystic lesions of the head and neck, excluding the thyroid. Acta Cytol. 1990;34(3):443-8.

16. Luiz RR, Costa AJL, Nadanovsky P. Epidemiologia e bioestatística na pesquisa odontológica. São Paulo: Atheneu; 2005.

17. Buckland JR, Manjaly G, Violaris N, Howlett DC. Ultrasoundguided cutting-needle biopsy of the parotid gland. J Laryngol Otol. 1999;113(11):988-92.

18 Smallman LA, Young JA, Oates J, Proops DW, Johnson AP. Fine needle aspiration cytology in the management ENT of patients. J Laryngol Otol. 1988;102(10):909-13.

19. Frable M, Frable WJ. Fine needle aspiration biopsy revisited. Laryngoscope. 1982;92(12):1414-8.

20. Young JE, Archibald SD, Shier KJ. Needle aspiration cytologic biopsy in head and neck masses. Am J Surg. 1981;142(4):484-9.

21. Wong DS, Wong LY. "Cystic" parotid swelling on FNA: significance on clinical management. Otolaryngol Head Neck Surg. 2004;130(5):593-6.
22. Kline TS. Handbook of fine needle aspiration biopsy cytology. $2^{\text {nd }}$ ed. New York: Churchill Livingstone; 1988.

23. Schwarz R, Chan NH, MacFarlane JK. Fine needle aspiration cytology in the evaluation of head and neck masses. Am J Surg. 1990;159 (5): 482-56.

Como citar este artigo:

Robaina TF, Coutinho ACA, Valladares CP, Dias EP, Lourenço SQC. Análise descritiva da correlação entre diagnósticos citopatológicos obtidos em punção aspirativa por agulha fina e diagnósticos histopatológicos de lesões orais. Rev Col Bras Cir. [periódico na Internet] 2007 Set-Out; 34(5). Disponível em URL: www.scielo.br/rcbc

Endereço para correspondência:

Tatiana Ferreira Robaina

Rua Jorge Rudge, n.89, ap. 1306

Vila Isabel

20550-220 - Rio de Janeiro - RJ

(0XX) $2139780961 \quad$ ou $\quad(0 X X) 2192803577$

tatirobaina@yahoo.com.br; tatianarobaina@gmail.com 